\title{
Fullerene nanoparticles ameliorate disease in arthritis mouse model
}

F ullerene derivatives can have antiinflammatory properties and have been shown to inhibit mast cell degranulation. In a new study published in PLoS ONE, Dellinger et al. show that amphiphilic liposomal malonylfullerene (ALM) and tetra-glycolic acid (TGA), two fullerene derivatives, decreased inflammation and ameliorated disease severity in a mouse model of arthritis.

$\mathrm{C}_{70}$ fullerenes are nanoparticles that can be modified with different moieties to improve their biological properties, and are capable of absorbing electrons and dispersing them through the $3 \mathrm{D} \pi$-conjugated structure distributed over their surface. "This antioxidant effect was one contributing factor that led us to research these nanomaterials as a potential nanomedicine for arthritis," says Anthony Dellinger, one of the authors of the study. Previous data had shown that some fullerene derivatives could stabilize human mast cells, one of the main immune cell subsets that infiltrate the inflamed synovia in patients with rheumatoid arthritis (RA). In the present study, the antiinflammatory and antioxidant potential of fullerene derivatives was assessed in vitro and in vivo using mouse models of RA.

From the panel of 40 water-soluble fullerene derivatives tested in vitro, $\sim 15 \%$ inhibited mast cell degranulation and IL- $1 \beta$ production, whereas $25 \%$ reduced IL- 6 and IL- 8 production by TNFstimulated human synovial fibroblasts. ALM and TGA, the two fullerene derivatives with the strongest capacity to inhibit cytokine production, also inhibited osteoclast differentiation from human peripheral blood mononuclear cells.
Mitochondrial membrane potential is closely associated with the degranulation process in mast cells, suggesting that the effect of fullerenes on degranulation might result from their antioxidant properties. In agreement with this hypothesis, preincubation of mast cells with ALM or TGA prevented the increase in mitochondrial membrane potential elicited after immune-complex stimulation of mast cells. Additionally, mast cells treated with ALM and TGA also showed reduced reactive oxygen species activity and NFkB expression.

ALM and TGA were then tested in vivo in the $\mathrm{K} / \mathrm{B} \times \mathrm{N}$ serum-transfer mouse model of arthritis, in which $\mathrm{C} 57 \mathrm{BL} / 6$ mice receive $\mathrm{K} / \mathrm{B} \times \mathrm{N}$ serum for disease induction. Treatment of arthritic mice with fullerene derivatives conjugated to IR-800 dye led to selective fullerene accumulation in inflamed joints of arthritic mice, but not in control mice, $6 \mathrm{~h}$ after injection of the conjugate. Importantly, treatment of arthritic mice with ALM and TGA led to decreased ankle and wrist thickness, reduced histopathology hallmarks of disease (including synovial hyperplasia, pannus formation and inflammatory infiltrates) and lowered TNF serum levels.

The protective effect of ALM and TGA on inflammatory arthritis was not dependent on mast cells, as fullerene treatment in mast-cell-deficient arthritic mice also decreased disease severity, albeit to a lesser extent when compared with mast-cell-sufficient mice. This finding suggests that the effects of fullerene derivatives on synovial fibroblasts and osteoclast differentiation found in vitro might play a part in the amelioration of disease observed in vivo. Furthermore, the arthritic disease observed in the $\mathrm{K} / \mathrm{B} \times \mathrm{N}$ mouse model seems independent of mast cells, because mast-cell-deficient mice still developed disease after $\mathrm{K} / \mathrm{B} \times \mathrm{N}$ serum transfer. Despite the protection conferred by ALM and TGA in the $\mathrm{K} / \mathrm{B} \times \mathrm{N}$ model, administration of fullerene derivatives to mice with collagen-

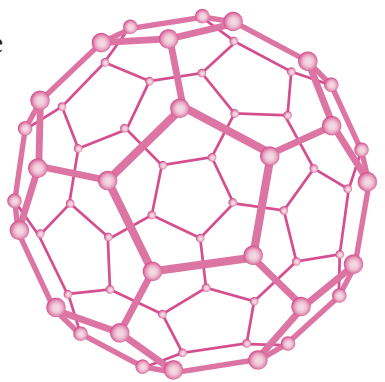
induced arthritis had no significant protective effect.

In the present study, administration of high concentrations of ALM and TGA to mice did not increase serum activity of alanine transaminase or aspartate aminotransferase, suggesting no overt liver toxicity. Previous studies assessing fullerene toxicity have yielded conflicting or inconclusive findings, as most did not use well-characterized and highly purified material. "Researchers must make significant strides to unravel toxicity conundrums," says Dellinger, adding that "extrapolation of results across such broad classes is not sufficient to characterize the toxicity profiles of these molecules."

According to Cameron Brown (University of Oxford, UK), who was not involved in this study, "fullerenes have been studied for a couple of decades and have generated a lot of interest in a number of fields; its application for scavenging of oxidant species is certainly exciting for musculoskeletal disease." The data presented by Dellinger et al. suggests a potential use of fullerenes as therapeutic agents in inflammatory arthritis, but further studies are needed before these findings can be translated to human disease.

João H. Duarte

Original article Dellinger, A. L. et al. Inhibition of inflammatory arthritis using fullerene nanomaterials. PLOS ONE 10, e0126290 (2015) 